\title{
Review of stochastic stability and analysis tumor-immune systems
}

Chiş Oana $^{* 1}$,Opriş Dumitru ${ }^{2}$ and Concu Riccardo ${ }^{3}$

${ }^{1}$ Instituto de Investigaciones Marinas, Spanish Council for Scientific Research (IIM-CSIC), C/Eduardo Cabello 6, Vigo, Spain

${ }^{2}$ West University of Timişoara, 4 Vasile Pârvan Blvd., Timişoara, 300223, Romania

${ }^{3}$ University of Porto, REQUIMTE-ICETA, Department of Chemistry and Biochemistry, Faculty of Sciences, Rua do Campo Alegre 687, 4169-007, Porto, Portugal

*Corresponding author: Chiş Oana, email: chisoana@yahoo.com

\begin{abstract}
In this paper we review and at the same time investigate some stochastic models for tumor-immune systems. To describe these models, we used a Wiener process, as the noise has a stabilization effect. Their dynamics are studied in terms of stochastic stability around the equilibrium points, by constructing the Lyapunov exponent, depending on the parameters that describe the model. Stochastic stability was also proved by constructing a Lyapunov function and the second order moments. We have studied and analyzed a Kuznetsov-Taylor like stochastic model and a Bell stochastic model for tumor-immune systems. These stochastic models are studied from stability point of view and they were graphically represented using the second order Euler scheme and Maple 12 software.
\end{abstract}

Keywords: stochastic models, Wiener process, stochastic stability, Lyapunov function, Lyapunov exponent.

\section{Introduction}

Stochastic modeling plays an important role in many branches of science. In many practical situations perturbations appear and these are expressed using white noise, modeled by Brownian motion. We will study stochastic dynamical systems that are used in medicine, in describing a tumor behavior, but still we do not know much about the mechanism of destruction and establishment of a cancer tumor, because a patient may experience tumor regression and later a relapse can occur. The need to address not only preventative measures, but also more successful treatment strategies is clear. Efforts along these lines are now being investigated through immunotherapy . This tumor-immune study, from theoretical point of view, has been done for two cell populations: effector cells and tumor cells. It was predicted a threshold above which 
there is uncontrollable tumor growth, and below which the disease is attenuated with periodic exacerbations occurring every 3-4 months. There was also shown that the model does have stable spirals, but the Dulac-Bendixson criterion demonstrates that there are no stable closed orbits. It is consider ordinary differential equations (ODE) for the populations of immune and tumor cells and it is shown that survival increases if the immune system is stimulated, but in some cases an increase in effector cells increases the chance of tumor survival.

In the last years, stochastic growth models for cancer cells were developed. We mention the papers of W.Y. Tan and C.W. Chen [1], N. Komarova, G. Albano and V.Giorno [2], L. Ferrante, S. Bompadre, L. Possati and L. Leone [3], and so on. Our goal in this paper is to construct stochastic models and to analyze their behavior around the equilibrium point. In these points stability is studied by analyzing the Lyapunov exponent, depending of the parameters of the models, Lyapunov function method and the square mean method. Numerical simulations are done using a deterministic algorithm with an ergodic invariant measure. In this work the authors studied and analyzed two stochastic models. In Section 2, we considered a Kuznetsov and Taylor stochastic model [4,5] (Subsection 2.1). Beginning from the deterministic model, we have studied the case of positive immune response. We gave the stochastic model and we analyzed its behavior in the equilibrium points. In Subsection 2.2 we presented a general family of tumorimmune stochastic systems and from this general representation we chose to analyze the Bell model. In Section 3 theoretical results are presented. We began with a stochastic dynamical system and we pointed out three methods for studying stability: stochastic moments method (Subsection 3.1), stochastic Lyapunov exponents (Subsection 3.2), and Lyapunov function method (Subsection 3.3). In Section 4 we have presented numerical simulation for Kuznetsov and Taylor model (Subsection 4.1), around an equilibrium point. We have illustrated the Lyapunov exponents and moments of the second order. In Subsection 4.2 we have considered the graphic representation for stochastic Bell model, and conclusions on stability were given, using the three methods: Lyapunov exponents, Lyapunov function and second order moments method $[5,6]$.

The initial model was written as a stochastic one, and its behavior around the equilibrium points was discused. We have proved stochastic stability around equilibrium point using three methods. The first one consists of constructing the second order moments, the second one expressing the Lyapunov exponent, and then drawing the conclusion when the considered system is stable. The 
third method is a way of constructing a Lyapunov function and determining sufficient conditions to guarantee the stability of the model. Numerical simulations were done using the software Maple 12 and the second order Euler scheme was implemented for a representation of the discussed stochastic models.

\section{Deterministic models}

\subsection{Kuznetsov and Taylor model}

The study of tumor-immune interaction is determined by the behavior of two populations of cells: effector cells and tumor cells. We will construct the stochastic models using well known deterministic models and we analyze stochastic stability around the equilibrium points. The analysis is done using Lyapunov exponent method. We begin our study from the deterministic model of Kuznetsov and Taylor [4]. This model describes the response of effector cells to the growth of tumor cells and takes into consideration the penetration of tumor cells by effector cells, that causes the interaction of those. This model can be represented in the following way:

$$
\left\{\begin{array}{l}
\dot{x}(t)=a_{1}-a_{2} x(t)+a_{3} x(t) y(t), \\
\dot{y}(t)=b_{1} y(t)\left(1-b_{2} y(t)\right)-x(t) y(y),
\end{array}\right.
$$

where initial conditions are $x(0)=x_{0}>0, y(0)=y_{0}>0$, and $a 3$ is the immune response to the appearance of the tumor cells. In this paper we consider the case of $a_{3}>0$, that means that immune response is positive. For the equilibrium states $P_{1}$ and $P_{2}$, we study the asymptotic behavior with respect to the parameter $a 1$ in (1). For $b_{1} a_{2}<a 1$, the system (1) has the equilibrium states $P_{1}\left(x_{1}, y_{1}\right)$ and $P_{2}\left(x_{2}, y_{2}\right)$, with

$$
\begin{gathered}
x_{1}=\frac{a_{1}}{a_{2}}, y_{1}=0, \\
x_{2}=\left(b_{1}\left(a_{3}-b_{2} a_{2}\right)+\sqrt{\Delta}\right) /\left(2 a_{3}\right), y_{2}=\left(b_{1}\left(a_{3}+b_{2} a_{2}\right)-\sqrt{\Delta}\right) /\left(2 b_{1} b_{2} a_{3}\right)
\end{gathered}
$$

where $\Delta=b_{1}^{2}\left(b_{2} a_{2}-a_{3}\right)^{2}+4 b_{1} b_{2} a_{1} a_{3}$. In [4] it is shown that there is an $a_{10}$, such that if $0<a_{1}<$ $a_{10}$, then the equilibrium state $P_{1}$ is asymptotically stable, and for $a_{1}>a_{10}$ the equilibrium state $P_{1}$ is unstable. If $a_{1}<a_{10}$, then the equilibrium state $P_{2}$ is unstable and for $a_{1}>a_{10}$ it is asymptotically stable. 


\subsection{A general family of tumor-immune systems}

A Volterra-like model was proposed in [7] for the interaction between a population of tumor cells (whose number is denoted by $x$ ) and a population of lymphocyte cells, $y$ and it is given by

$$
\left\{\begin{array}{l}
\dot{x}(t)=a x(t)-b x(t) y(t), \\
\dot{y}(t)=d x(t) y(t)-f x(t)-k x(t)+u,
\end{array}\right.
$$

where the tumor cells are supposed to be in exponential growth (which is, however, a good approximation only for the initial phases of the growth) and the presence of tumor cells implies a decrease of the "input rate" of lymphocytes. A general representation for such models can be considered in the form given by d'Onofrio in [8]:

$$
\left\{\begin{array}{c}
x(t)=f_{1}(x(t), y(t)), \dot{y}(t)=f_{2}(x(t), y(t)), \\
x(0)=x_{0}, y(0)=y_{0},
\end{array}\right.
$$

where $x$ is the number of tumor cells, $y$ the number of effector cells of immune system and

$$
\begin{aligned}
& f_{1}(x, y)=x\left(h_{1} x-h_{2} y\right), \\
& f_{2}(x, y)=\left(h_{3} x-h_{4} x\right) y+h_{5} x .
\end{aligned}
$$

The functions $h_{1}, h_{2}, h_{3}, h_{4}, h_{5}$ are given such that the system (5) admits the equilibrium point $P_{1}\left(x_{1}, y_{1}\right)$, with $x_{1}=0, y_{1}>0$, and $P_{2}\left(x_{2}, y_{2}\right)$, with $x_{1} \neq 0, y_{2}>0$. The analysis of these models was proven also using numerical simulations. Deterministic models of this general form are the following:

\section{Volterra model [9] if}

$h_{1}(x)=a_{1}, h_{2}(x)=a_{2} x, h_{3}(x)=b_{3} x, h_{4}(x)=b_{2}, h_{5}(x)=-b_{1} x$,

\section{Bell model}

$h_{1}(x)=a_{1} x, h_{2}(x)=a_{1} x, h_{3}(x)=b_{1} x, h_{4}(x)=b_{3}, h_{5}(x)=-b_{2} x+b_{4}$,

Stepanova model [10] with

$h_{1}(x)=a_{1}, h_{2}(x)=1, h_{3}(x)=b_{1} x, h_{4}(x)=b, h_{5}(x)=-b_{2} x+b_{4}$,

Vladar-Gonzalez model [11] if in (5) we consider

$$
h_{1}(x)=\log (K / x), h_{2}(x)=1, h_{3}(x)=b_{1} x, h_{4}(x)=b_{2}+b_{3} x^{2}, h_{5}(x)=1,
$$

Exponential model [12] if in (5) we consider

$$
h_{1}(x)=1, h_{2}(x)=1, h_{3}(x)=b_{1} x, h_{4}(x)=b_{2}+b_{3} x^{2}, h_{5}(x)=1,
$$


Logistic model [13] if in (5) we consider

$h_{1}(x)=1-\frac{a_{1}}{x}, h_{2}(x)=1, h_{3}(x)=b_{1} x, h_{4}(x)=b_{2}+b_{3} x^{2}, h_{5}(x)=1$.

\section{Stochastic models and associated moments}

In this section we construct the stochastic models, in the general case, by using two approaches, depending on the diffusion function. For a two dimensional dynamical system, we will consider the diffusion function expressed as a matrix, for determining the Lyapunov exponents, and, without restricting the generality, but for a better computation, we will consider the diffusion expressed as diagonal matrix, for determining the associated stochastic moments.

\subsection{Stochastic moments}

Let $\left(\Omega, F_{t \geq 0}, P\right)$ be the given probability space, and $(W(t))_{t \geq 0}$ a standard Wiener process adapted to the filtration $(F)_{t \geq 0}$ and having independent stationary Gaussian increments with $W(0)=0$, $E(W(t)-W(s))=0$ and $E(W(t) W(s))=\min (t, s)$, where $E$ denotes mathematical expectation [14]. The sample trajectories of $W(t)$ are continuous, nowhere differentiable and have infinite variation on any finite time interval. Linearizing the dynamical systems (2.1) and (2.5) around the equilibrium point $\left(x_{0}, y_{0}\right)$, we get the linear differential equation

$$
\dot{z}(t)=A z(t)
$$

where $z(t)=\left(z_{1}(t), z_{2}(t)\right)^{T}$ and $A=\left(a_{i j}\right)=\left.\frac{\partial f_{i}}{\partial x_{j}}\right|_{\left(x_{0}, y_{0}\right)}, i, j=1,2$ and the linear stochastic differential equations with $B=\operatorname{diag}\left(\sigma_{1}, \sigma_{2}\right)$.

$$
d z(t)=A z(t) d t+B z(t) d W(t),
$$

A solution for stochastic dynamical system (8) is given by

$$
z(t)=z(0)+\int_{0}^{t} A z(s) d s+\int_{0}^{t} B z(s) d W(s) .
$$

The solution is a stochastic process denoted by $z(t)=z(t, \omega)$. From the Chebyshev inequality, the possible rang of $z$ at a time $t$ is "almost" determined by its mean and variance at time $t$. So, the first and second moments are important for investigating the solution behavior.

Proposition 3.1 [15] The moments of the solution of (8) is given by

$$
\frac{d E(z(t))}{d t}=A E(z(t))
$$


The proof results by taking the mathematical expectation on both sides of (8), and from Itô integral properties. The stability of the second moments of $z(t)$, for the stochastic equation (8), can be examined by using Itô's rule for the given stochastic equation of $z(t) z^{T}(t)$.

$$
\frac{d}{d t} E\left(z(t) z^{T}(t)\right)=E\left\{d z(t) d z^{T}(t)+z(t) d z^{T}(t)+B z(t) z^{T}(t) B\right\}=E\left\{A z(t) z^{T}(t)+z(t) z^{T}(t) A^{T}+B z(t) z^{T}(t)\right\}
$$

Let $R(t, s)=E\left\{z(t) z^{T}(t)\right\}$ be the covariance matrix of the process $z(t)$ such that $R(t, t)$ satisfies the following relation

$$
\dot{R}(t, t)=A R(t, t)+R(t, t) A^{T}+B R(t, t) B
$$

From (10) results

Proposition 3.2 The system of differential equations (10) is given by

$$
\begin{aligned}
& \dot{R}_{11}(t, t)=\left(2 a_{11}+\sigma_{1}^{2}\right) R_{11}(t, t)+2 a_{12} R_{12}(t, t), \\
& \dot{R}_{22}(t, t)=\left(2 a_{22}+\sigma_{2}^{2}\right) R_{22}(t, t)+2 a_{21} R_{12}(t, t), \\
& \dot{R}_{12}(t, t)=a_{21} R_{11}(t, t)+a_{12} R_{22}(t, t)+\left(a_{11}+a_{22}+\sigma_{1} \sigma_{2}\right) R_{12}(t, t),
\end{aligned}
$$

with $R_{i j}(t, t)=E\left(z_{i}(t) z_{j}(t)\right), i, j=1,2$;

2. The characteristic function of (11) is given by

$$
f(\lambda)=\left(\begin{array}{ccc}
2 \lambda-2 a_{11}-\sigma_{1}^{2} & 0 & 2 a_{12} \\
0 & 2 \lambda-2 a_{22}-\sigma_{2}^{2} & 2 a_{21} \\
2 a_{21} & 2 a_{12} & 2 \lambda-a_{11}-a_{22}-\sigma_{1} \sigma_{2}
\end{array}\right)
$$

\subsection{Stochastic Lyapunov exponents}

Stochastic stability can be investigated by constructing the Lyapunov exponents [5]. If the Lyapunov exponent is negative, then the dynamical system is stabile. This study is done around an equilibrium point and the linearized of the stochastic dynamical system (8). From the Oseledec multiplicative ergodic theorem [16], there are two nonrandom Lyapunov exponents $\lambda_{2} \leq \lambda_{1}=\lambda$ where $\lambda$ is given by:

$$
\lambda=\lim _{t \rightarrow \infty} \sup \frac{1}{t} \log \sqrt{z_{1}(t)^{2}+z_{2}(t)^{2}} .
$$

By applying a change of coordinate

$$
u_{1}(t)=r(t) \cos \theta(t), \quad u_{2}(t)=r(t) \sin \theta(t)
$$


for (8), and by using the Itô formula, we get the integral form of the stochastic equations, given by

$$
\begin{aligned}
& \log \frac{r(t)}{r(0)}=\int_{0}^{t} q_{1}(\theta(s))+\frac{1}{2}\left(q_{4}(\theta(s))^{2}-q_{2}(\theta(s))^{2}\right) d s+\int_{0}^{t} q_{2}(\theta(s)) d W(s) \\
& \left.\theta(\mathrm{t})=\theta(0)+\int_{0}^{t} q_{3}(\theta(s))-q_{2}(\theta(s)) q_{4}(\theta(s))\right) d s+\int_{0}^{t} q_{4}(\theta(s)) d W(s)
\end{aligned}
$$

where

$$
\begin{aligned}
& q_{1}(\theta)=a_{11} \cos ^{2} \theta+\left(a_{12}+a_{21}\right) \cos \theta \sin \theta+a_{22} \sin ^{2} \theta, \\
& q_{2}(\theta)=b_{11} \cos ^{2} \theta+\left(b_{12}+b_{21}\right) \cos \theta \sin \theta+b_{22} \sin ^{2} \theta, \\
& q_{3}(\theta)=a_{21} \cos ^{2} \theta+\left(a_{22}-a_{11}\right) \cos \theta \sin \theta-a_{12} \sin ^{2} \theta \\
& q_{4}(\theta)=b_{21} \cos ^{2} \theta+\left(b_{22}-b_{11}\right) \cos \theta \sin \theta-b_{12} \sin ^{2} \theta
\end{aligned}
$$

As the expectation of the Itô stochastic integral is null, the Lyapunov exponent is given by

$$
\lambda=\lim _{t \rightarrow \infty} \frac{1}{t} \log \frac{r(t)}{r(0)} \text {. }
$$

If $r(t)$ is ergodic, it results that

$$
\lambda=\int_{0}^{t}\left(q_{1}(\theta)+\frac{1}{2}\left(q_{4}(\theta)^{2}-q_{2}(\theta)\right)\right) p(\theta) d \theta
$$

where $p(\theta)$ is the probability density of the process $\theta$.

\subsection{Lyapunov function}

Another manner to determine whether a solution is stable or not is by constructing a Lyapunov function. This function gives conditions for stability. In general, the drift and diffusion functions that appear in the description of a stochastic dynamical system are hard to use, because they are nonlinear. That is why we will work with the linearized system. Let consider the differential operator given by

$$
L V(t, x)=\frac{\partial V(t, x)}{\partial t}+\sum_{i=1}^{2} f_{i}(x) \frac{\partial V(t, x)}{\partial x_{i}}+\frac{1}{2} \sum_{i=1}^{2} \sum_{j=1}^{2} g_{i}(x) g_{j}(x) \frac{\partial^{2} V(t, x)}{\partial x_{i} \partial x_{j}}
$$

a definition domain $D=\left\{(t \geq 0) \times \mathbb{R}^{2}\right\}$ and $V: D \rightarrow \mathbb{R}$ a continuous function with respect to $t$ and of the class $C^{2}$ with respect to the other components. Then we have the following results that we will use in the following.

Theorem 3.3 If the function $V: D \rightarrow \mathbb{R}$ satisfies the inequalities

$$
\begin{gathered}
k_{1}\|u\|^{p} \leq V(t, u) \leq k_{2}\|u\|^{p}, \\
L V(t, u) \leq-k_{3}\|u\|^{p}, \quad k_{i}>0, p>0,
\end{gathered}
$$

then the trivial solution of (8) is exponentially p-stable for $t \geq 0$. 
In concrete problems, the next theorem is used.

Theorem 3.4 If the function $V: D \rightarrow \mathbb{R}$ satisfies

(i) $L V(u) \leq 0$, then the trivial solution is stable in probability;

(ii) $L V(u) \leq-c(\|u\|)$, where $c: \mathbb{R}_{+} \rightarrow \mathbb{R}_{+}$is a continuous function, then the trivial solution is asymptotically stable;

(iii) $L V(u) \leq q^{T} Q q$, where $Q$ is a symmetric matrix, positive defined, then the trivial solution is stable in mean square value.

\section{The analysis of associated stochastic models. Numerical simulation.}

We associate a stochastic system of differential equations to the ordinary system of differential equations (1). Let us consider $\left(\Omega, F_{t \geq 0}, P\right)$ be the filtered probability space, and $(W(t))_{t \geq 0}$ a standard Wiener process adapted to the filtration $(F)_{t \geq 0}$. Let $\{X(t, \omega)=(x(t), y(t))\}_{t \geq 0}$ be a stochastic process. The system of Itô equations associated to system (1) is given by

$$
\left\{\begin{array}{l}
x(t)=x_{0}+\int_{0}^{t}\left(a_{1}-a_{2} x(s)+a_{3} x(s) y(s)\right) d s+\int_{0}^{t} g_{1}(x(s), y(s)) d W(s), \\
y(t)=y_{0}+\int_{0}^{t}\left(b_{1} y(s)\left(1-b_{2} y(s)\right)-x(s) y(s)\right) d s+\int_{0}^{t} g_{2}(x(s), y(s)) d W(s),
\end{array}\right.
$$

and the system of Itô equations associated to system (5) is

$$
\left\{\begin{array}{c}
x(t)=x_{0}+\int_{0}^{t}\left[x(s)\left(h_{1}(x(s))-h_{2}(x(s)) y(s)\right] d s+\int_{0}^{t} g_{1}(x(s), y(s)) d W(s),\right. \\
y(t)=y_{0}+\int_{0}^{t}\left[h_{3}(x(s))-h_{4}(x(s)) y(s)+h_{5}(x(s))\right] d s+\int_{0}^{t} g_{2}(x(s), y(s)) d W(s),
\end{array}\right.
$$

where, in both cases, the first integral is a Riemann integral, and the second one is an Itô integral. $\{\mathrm{W}(\mathrm{t})\}_{\mathrm{t}>0}$ is a Wiener process. The functions $g_{1}(x(t), y(t))$ and $g_{2}(x(t), y(t))$ are given in the case when we are working in the equilibrium state. The functions $g_{1}(x(t), y(t))$ and $g_{2}(x(t), y(t))$ represent the volatilizations of the stochastic equations and they are the therapy test functions. In $P_{1}$ those functions have the following form

$$
\begin{aligned}
& g_{1}(x(t), y(t))=b_{11} x(t)+b_{12} y(t)+c_{11}, \\
& g_{2}(x(t), y(t))=b_{21} x(t)+b_{22} y(t)+c_{21},
\end{aligned}
$$

where

$$
c_{11}=-b_{11} x_{1}-b_{12} y_{1}, c_{21}=-b_{21} x_{1}-b_{22} y_{1} \text {, }
$$

In the equilibrium state $P_{2}$, the functions $g_{1}(x(t), y(t))$ and $g_{2}(x(t), y(t))$ are given by

$$
\begin{gathered}
g_{1}(x(t) ; y(t))=b_{11} x(t)+b_{12} y(t)+c_{12}, \\
g_{2}(x(t) ; y(t))=b_{21} x(t)+b_{22} y(t)+c_{22},
\end{gathered}
$$

where 


$$
c_{12}=-b_{11} x_{2}-b_{12} y_{2}, c_{22}=-b_{21} x_{2}-b_{22} y_{2} .
$$

\subsection{Kuznetsov and Taylor stochastic models}

First of all, we will investigate the stability of the stochastic system (19), by using the Lyapunov exponents method [5]. The linearized stochastic dynamical system (8), for Kuznetsov and Taylor stochastic model [4], is expressed using the matrices $A$ and $B$, in the equilibrium point $P_{1}\left(\frac{a_{1}}{a_{2}}, 0\right)$ by

$$
A=\left(\begin{array}{cc}
-a_{2}+a_{3} y_{1} & a_{3} x_{1} \\
-y_{1} & b_{1}-2 b_{2} y_{1}-x_{1}
\end{array}\right), \quad B=\left(\begin{array}{cc}
\alpha & -\beta \\
\beta & \alpha
\end{array}\right)
$$

For numerical simulations we use the following values of parameters: $a_{1}=0.1181, a_{2}=0.3747$, $a_{3}=0.01184, b_{1}=1.636 b_{2}=0.002$ and $\alpha=10, \beta=2$. In a similar way, matrices $A$ and $B$ are defined in the second equilibrium point; with $\Delta=b_{1}^{2}\left(b_{2} a_{2}-a_{3}\right)^{2}+4 b_{1} b_{2} a_{1} a_{3}$. Using second order Euler scheme, for the ODE system (1) and SDE system (19), in the equilibrium point $P_{1}$, we get the following orbits presented in the figures above (see Figure1-Figure 6).

$$
P_{2}\left(\frac{\left(-b_{1}\left(b_{2} a_{2}-a_{3}\right)+\sqrt{\Delta}\right.}{2 a_{3}}, \frac{\left(b_{1}\left(b_{2} a_{2}+a_{3}\right)-\sqrt{\Delta}\right.}{2 b_{1} b_{2} a_{3}}\right)
$$

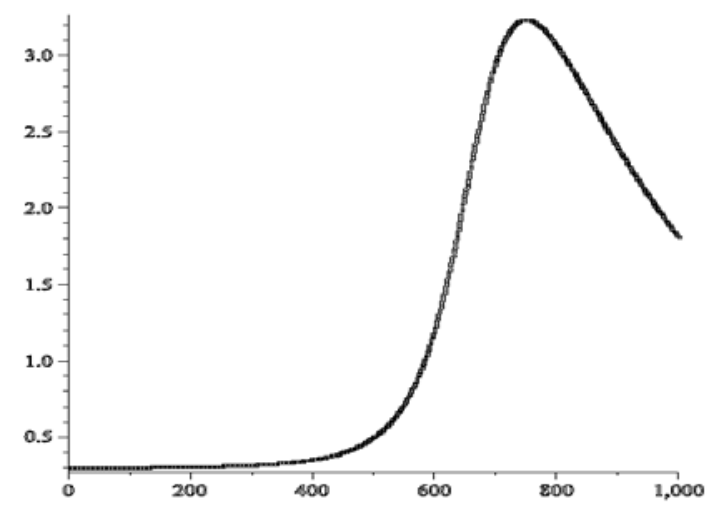

Figure 1: (n,x(n)) in $P_{1}$ for ODE (1)

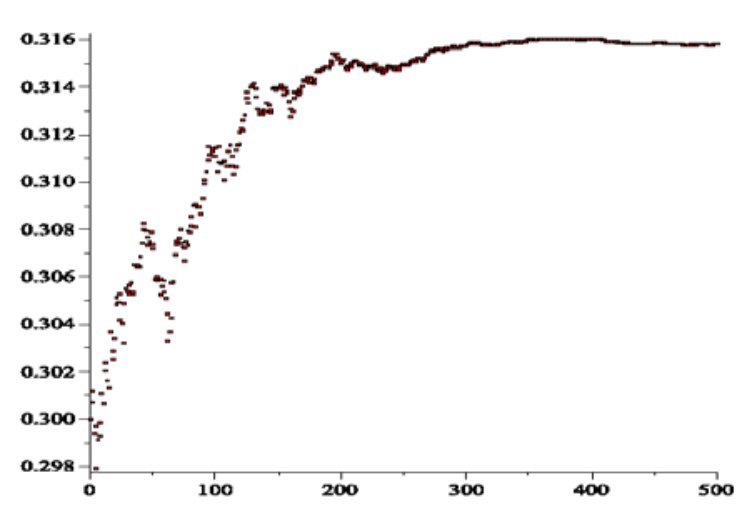

Figure 2: $(\mathrm{n}, \mathrm{x}(\mathrm{n}, \omega))$ in $P_{1}$ for SDE (19) 

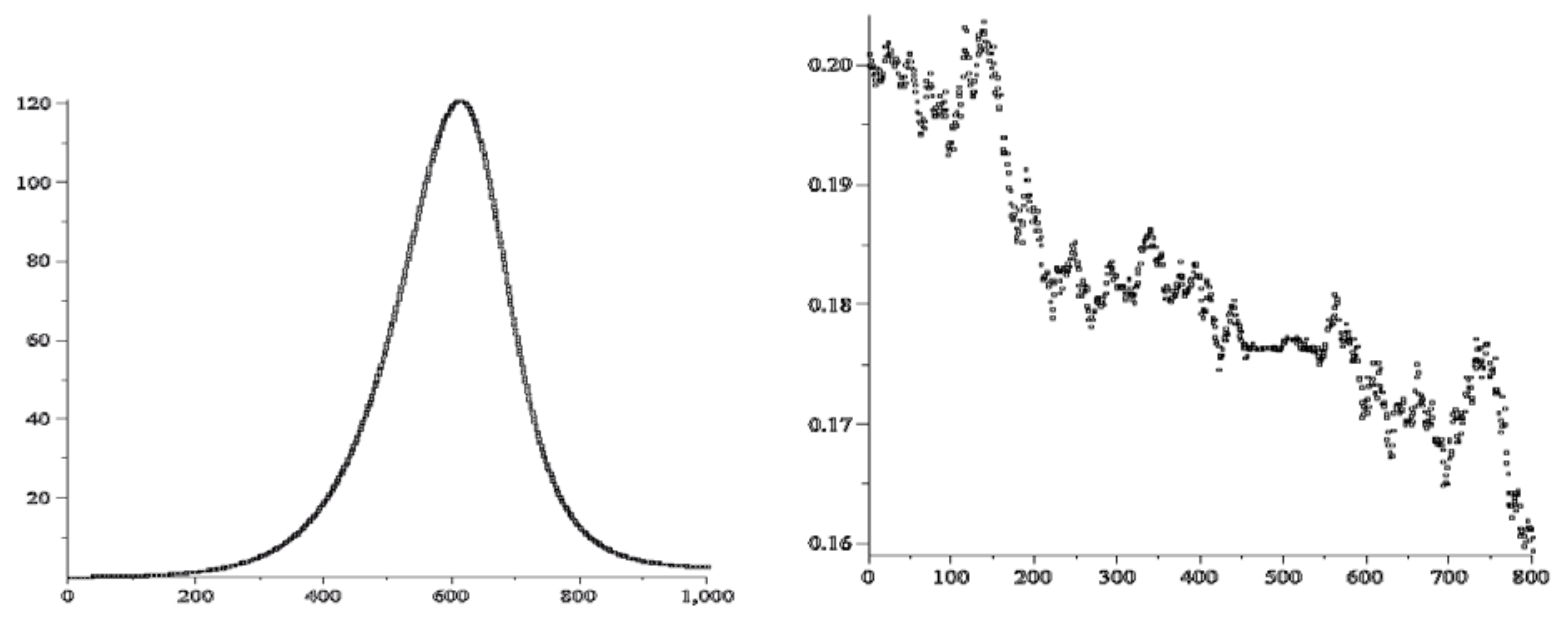

Figure 3: (n,y(n)) in $P_{1}$ for ODE (1)

Figure 4: $(\mathrm{n}, \mathrm{y}(\mathrm{n}, \omega))$ in $P_{1}$ for SDE (19)
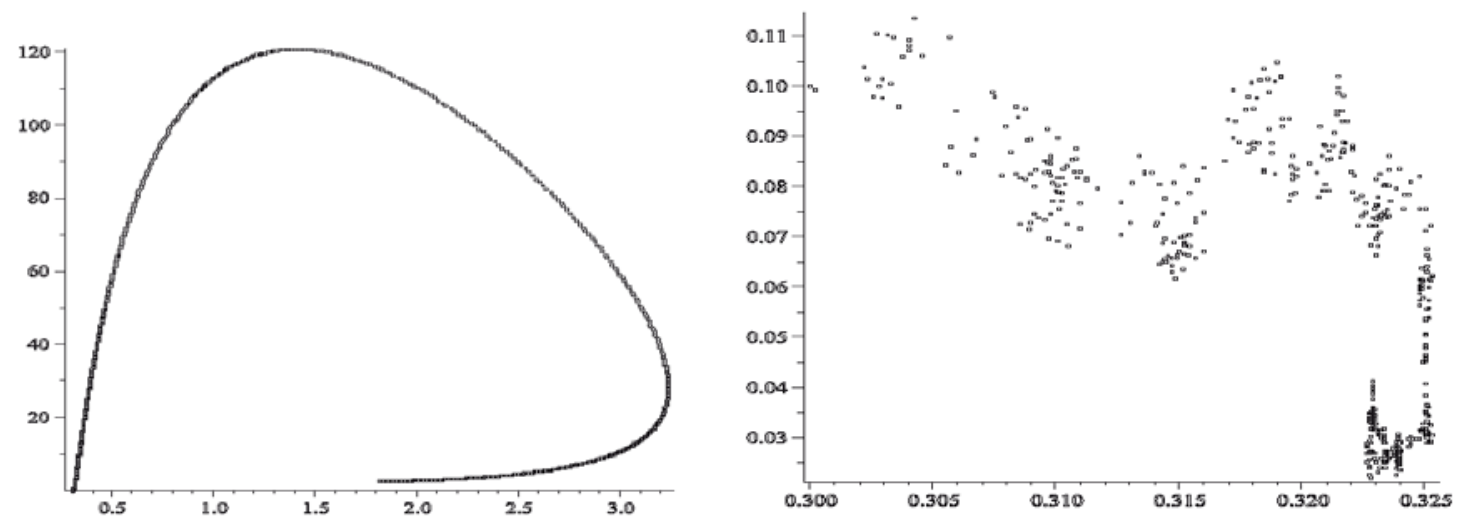

Figure 5: $(x(n), y(n))$ in $P_{1}$ for ODE (1)

Figure 6: $(\mathrm{x}(\mathrm{n}, \omega), \mathrm{y}(\mathrm{n}, \omega))$ in $P_{1}$ for SDE (19) The Lyapunov exponent variation, with the variable parameter $b_{11}=\alpha$, is given in Figure 7, respectively Figure 8 , for $P_{1}$, respectively $P_{2}$. 


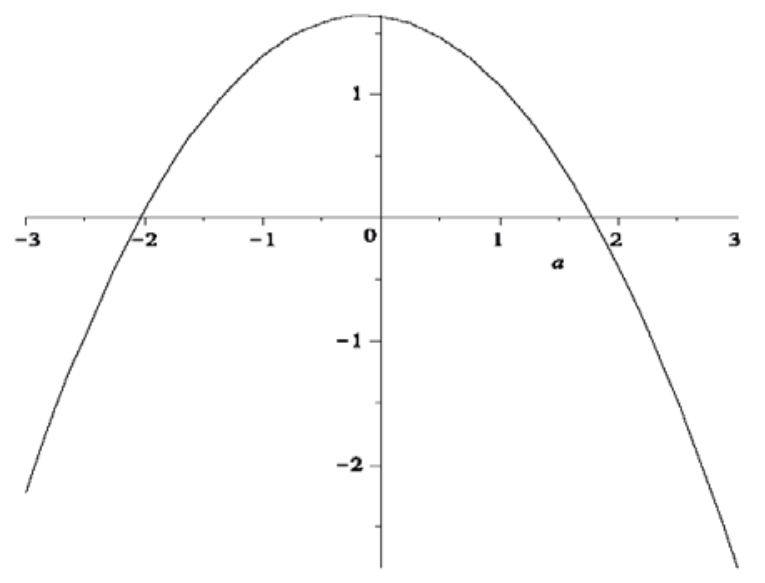

Figure 7: $(\alpha, \lambda(\alpha))$ in $P_{1}$ for ODE (1)

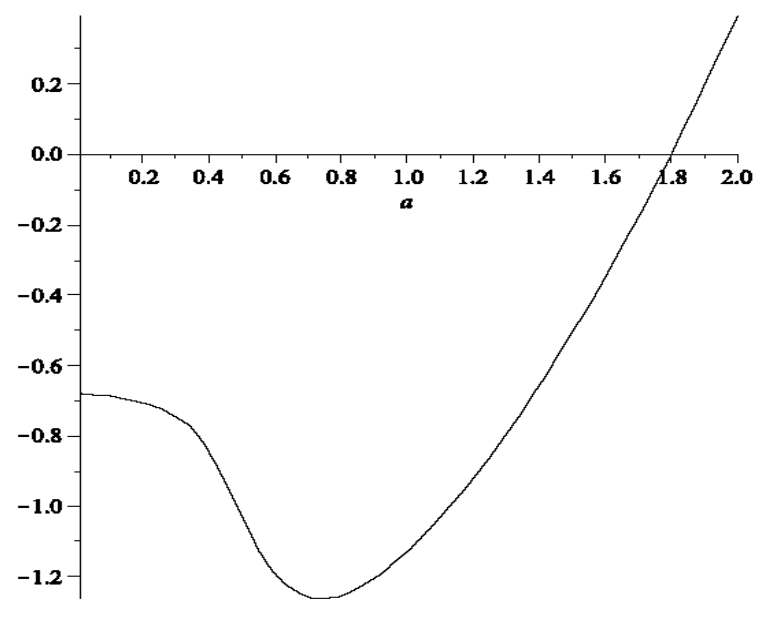

Figure $8(\alpha, \lambda(\alpha))$ in $P_{2}$ for SDE (19)

The Lyapunov exponent, for the equilibrium point $P_{1}$ is negative, so $P_{1}$ is asymptotically stable for each $\alpha \in \mathbb{R}$. The second equilibrium point $P_{2}$ is asymptotically stable for all values when $\lambda<$ 0 , that means that $P_{2}$ is unstable for all $\alpha \in(-\infty,-1.8) \cup(1.8, \infty)$. Stochastic moments of the second order (Figure9 - Figure11) and the dispersions (Figure 12 and figure 13) are calculated using the method presented in Section 3.1. We will begin from the linearized dynamical system (8), expressed in terms of the matrices $A$ and $B$ given above, in the equilibrium point $P_{1}\left(\mathrm{a}_{1} / \mathrm{a}_{2}, 0\right)$. For the numerical simulation we will use the following values of the parameters: $a_{1}=7.81, a_{2}=$ 3.747, $a_{3}=0.1184, b_{1}=1.636, b_{2}=0.002$, and $\beta=0, \alpha=\sigma_{1}=\sigma_{2}=-1$. For the Kuznetsov and Taylor model, in the equilibrium point $P_{1}$ the characteristic function for differential equation for square root mean is given by (12)

$$
f(\lambda)=\left(\begin{array}{ccc}
2 \lambda+2 a_{2}-\sigma_{1}^{2} & 0 & 2 \frac{a_{1} a_{3}}{a_{2}} \\
0 & 2 \lambda-2\left(a_{1}-\frac{a_{1}}{a_{2}}\right)-\sigma_{2}^{2} & 0 \\
0 & \frac{a_{1} a_{3}}{a_{2}} & 2 \lambda+a_{2}-b_{1}+\frac{a_{1}}{a_{2}}-\sigma_{1} \sigma_{2}
\end{array}\right) .
$$


For the above values of the parameters, results that $P_{1}$ is stable, as the eigenvalues of the matrix $A$ in $P_{1}$ have real parts less than zero, and the moments of the second order are unstable, because (12) has a positive solution.
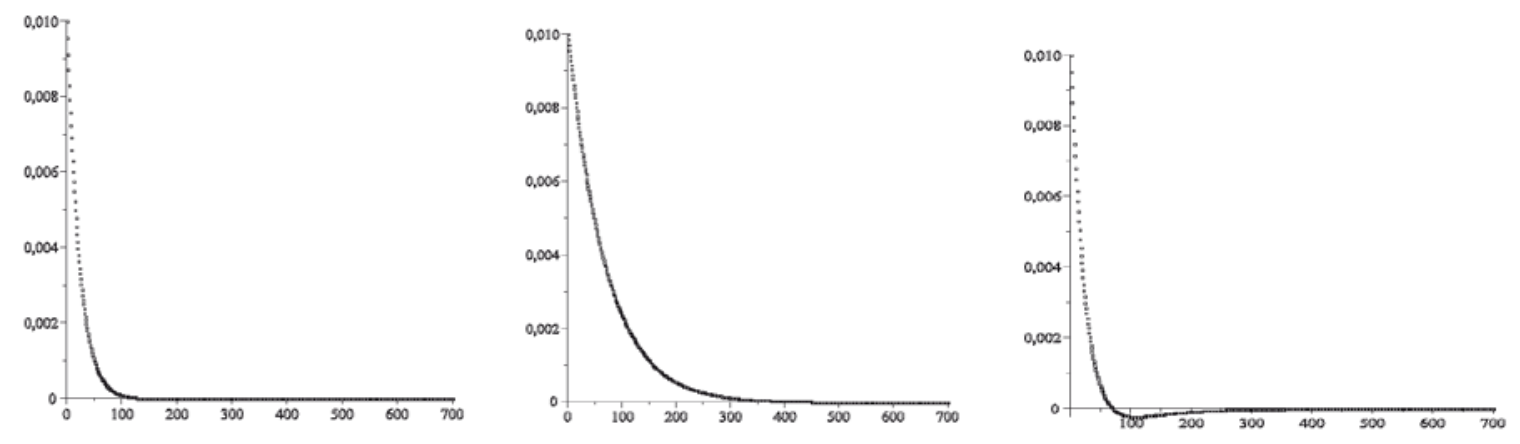

Figure 9: (n, $\left.\mathrm{R}_{11}(\mathrm{n})\right)$

Figure 10: $\left(n, R_{22}(n)\right)$

Figure 11: $\left(\mathrm{n}, \mathrm{R}_{12}(\mathrm{n})\right)$

The orbits for dispersions, $(n, D(1,1)(n))$, $(n, D(2,2)(n))$, where $D(i, i)=R(i, i)-E(i, i)^{2}$, are represented in the figures below.
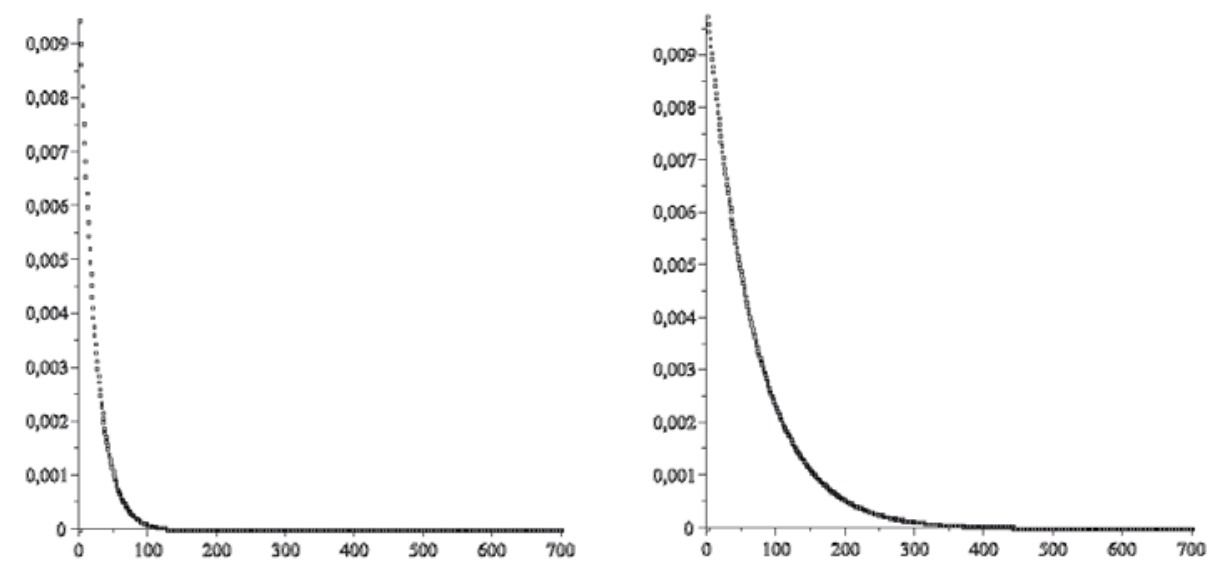

Figure 12: $(\mathrm{n}, \mathrm{D}(1,1)(\mathrm{n}))$

Figure 13: $(\mathrm{n}, \mathrm{D}(2,2)(\mathrm{n}))$ 


\subsection{Analysis of Bell model. Numerical simulations.}

Following the algorithm for determining the Lyapunov exponent and the description of second order Euler scheme in Maple 12 software, we get the following results, illustrated in the figures below. The orbits of ODE system (1) and SDE system (19), in the equilibrium point $P_{2}$ are presented in Figure14-Figure 17.

For numerical simulations we use the following values of parameters: $a_{1}=2.5, a_{2}=1, b_{1}=1, b_{2}$ $=0.4, b_{3}=0.95, b_{4}=2$. The matrices $A$ and $B$ are given, in the equilibrium point $P_{2}\left(\frac{a_{1} b_{3}-a_{2} b_{4}}{a_{1} b_{1}-a_{2} b_{2}}, \frac{a_{1}}{a_{2}}\right)$ by with $\alpha=a \in \mathbb{R}, \beta=-2$.

In a similar way the matrices $A$ and $B$ are defined in the other equilibrium point.

$$
A=\left(\begin{array}{cc}
-a_{2} y_{2}+a_{1} & -a_{2} x_{2} \\
-b_{2}+b_{1} y_{2} & b_{1} x_{2}-b_{3}
\end{array}\right), \quad B=\left(\begin{array}{cc}
\alpha & -\beta \\
\beta & \alpha
\end{array}\right),
$$
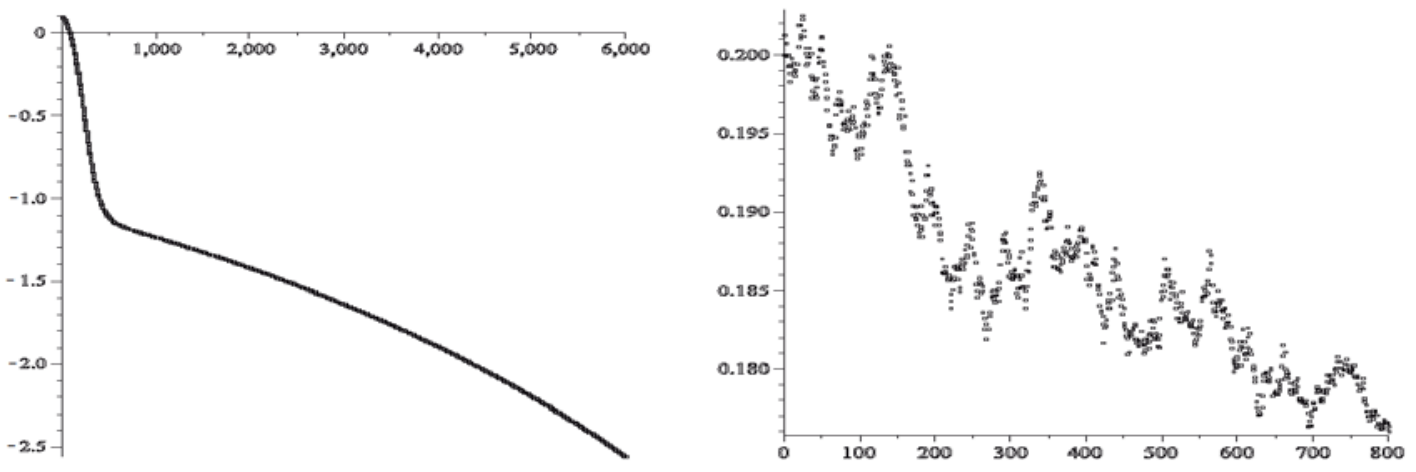

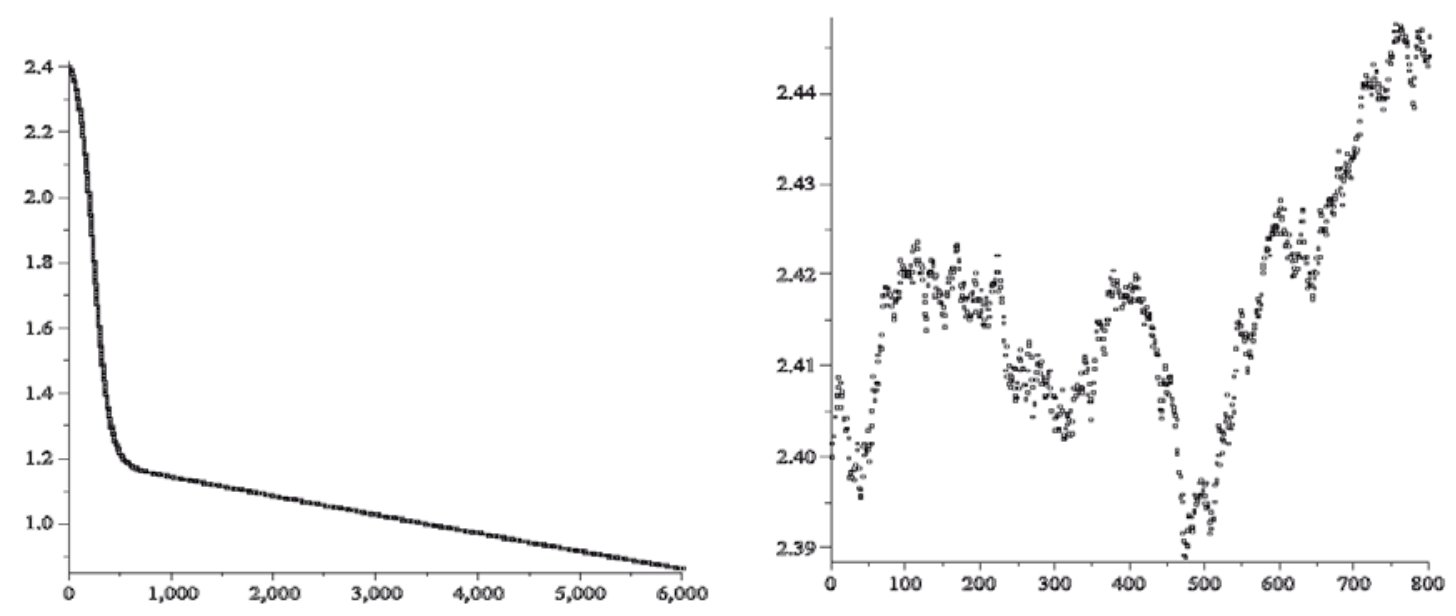

Figure 16: $(\mathrm{n}, \mathrm{y}(\mathrm{n}))$ in $\mathrm{P}_{2}$ for ODE (5)

Figure 17: $(\mathrm{n}, \mathrm{y}(\mathrm{n}, \omega))$ in $\mathrm{P}_{2}$ for SDE (20)

The variation of Lyapunov exponent with the variable parameter $b_{11}=\alpha$ is given in Figure 18 for $P_{1}$ and in Figure 19 for $P_{2}$.

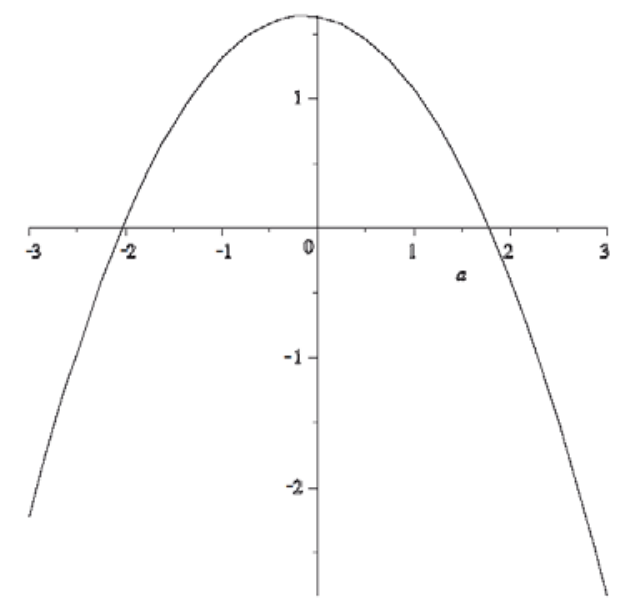

Figure 18: $(\alpha, \lambda(\alpha))$ in $\mathrm{P}_{1}$ for ODE (5)

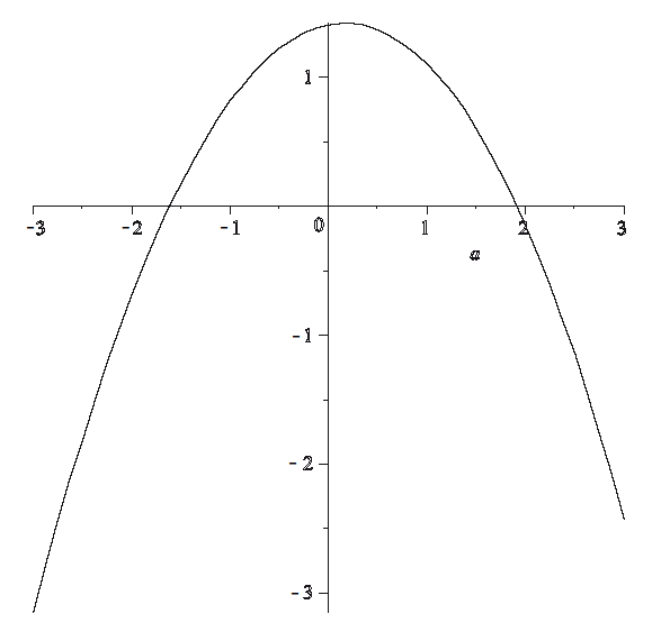

Figure 19: $(\alpha, \lambda(\alpha))$ in $\mathrm{P}_{2}$ for SDE (20) 
From the figures above, the equilibrium points $P_{1}$ and $P_{2}$ are asymptotically stable for all $\alpha$ such that the Lyapunov exponents $\lambda(\alpha)<0$, and unstable otherwise. So, $P_{1}$ is asymptotically stable for $\alpha \in(-\infty,-2.02) \cup(1.78, \infty)$ and $P_{2}$ is asymptotically stable for $\alpha \in(-\infty,-1.62) \cup$ $(1.88, \infty)$. Using Lyapunov function method [16], for the system of differential equations that describes Bell model, the next assertions are true.

Proposition 4.1 (a) The matrix of the system of differential equations that describes the linearized in $P_{2}$ is $A=\left(\begin{array}{cc}0 & a_{12} \\ a_{21} & a_{22}\end{array}\right)$,

where $a_{12}=\frac{a_{2}\left(a_{1} b_{3}-a_{2} b_{4}\right)}{a_{1} b_{1}-a_{2} b_{2}}, a_{21}=\frac{a_{1} b_{1}-a_{2} b_{2}}{a_{2}}, a_{12}=\frac{a_{2}\left(b_{1} b_{4}+b_{2} b_{3}\right)}{a_{1} b_{1}-a_{2} b_{2}}$;

(b) If $a_{1} b_{1}-a_{2} b_{2}>0$ and $a_{1} b_{3}-a_{2} b_{4}>0$, then the equilibrium point $P_{2}$ is asymptotically stable.

The stochastic model is given using a perturbation around the equilibrium point $P_{2}\left(x_{2}, y_{2}\right)$, in the following way with $\sigma_{1}, \sigma_{2}>0$.

$$
\left\{\begin{array}{l}
d x(t)=\left(x(t)-a_{2} y(t)\right) d t+\sigma_{1}\left(x(t)-x_{P_{2}}\right) d W^{1}, \\
d y(t)=\left[\left(b_{1} x(t)-b_{3}\right) y(t)-b_{2} x(t)+b_{4}\right] d t+\sigma_{2}\left(y(t)-y_{P_{2}}\right) d W^{2},
\end{array}\right.
$$

The linearized of system $(25)$ in $(0,0)$ is given by

$$
d u(t)=h(u(t)) d t+l(u(t)) d W(t)
$$

where $u(t)=\left(u_{1}(t), u_{2}(t)\right)^{T}, W(t)=\left(W^{1}(t), W^{2}(t)\right)^{T}$ and

$$
\begin{gathered}
h(u(t))=\left(\begin{array}{c}
\left(a_{1}-a_{2} y_{2}\right) u_{1}(t)-x_{2} a_{2} u_{2}(t) \\
\left(b_{1} y_{2}-b_{2}\right) u_{1}(t)+\left(b_{1} x_{2}-b_{3}\right) u_{2}(t)
\end{array}\right), \\
l(u(t))=\left(\begin{array}{cc}
\sigma_{1} u_{1}(t) & 0 \\
0 & \sigma_{2} u_{2}(t)
\end{array}\right) .
\end{gathered}
$$

We consider the set $D=\left\{(t \geq 0) \times \mathbb{R}^{2}\right\}$ and $V: D \rightarrow \mathbb{R}$ a function of class $C^{1}$ with respect to $t$, and of class $C^{2}$ with respect to the other variables. We study the $p$-exponential stability of the solution $(0,0)$ of the linearized stochastic system (26). Using Theorem 5.4, for the function $V: D \rightarrow \mathbb{R}$ we get the following result.

$$
V(t, u)=\frac{1}{2}\left(\omega_{1} u_{1}^{2}+\omega_{12} u_{2}^{2}\right), \quad \omega_{1}, \omega_{2} \in R_{+}
$$


Proposition 4.2 If the following relations take place

$$
\begin{gathered}
q_{1}=\omega_{1}\left(a_{2} y_{2}-a_{1}-\sigma_{1}^{2}\right)>0, q_{2}=\omega_{2}\left(b_{3}-b_{1} y_{2}-\sigma_{2}^{2}\right)>0, \\
b_{1} y_{2}-b_{2}>0, \omega_{1}=\frac{b_{1} y_{2}-b_{2}}{a_{2} y_{2}} \omega_{2},
\end{gathered}
$$

then $d V(t, u)=-u(t)^{T} Q u(t)$, with $Q$ given by $Q=\left(\begin{array}{cc}q_{1} & 0 \\ 0 & q_{2}\end{array}\right)$.

The equilibrium point of (25) is asymptotically stable in quadratic square $(p=2)$.

Proof: From (27), (28) and (29), we get

$$
\begin{gathered}
d V(t, u)=\left(\begin{array}{c}
\left(a_{1}-a_{2} y_{2}\right) u_{1}-x_{2} a_{2} u_{2} \\
\left(b_{1} y_{2}-b_{2}\right) u_{1}+\left(b_{1} x_{2}-b_{3}\right) u_{2}
\end{array}\right)^{T}\left(\begin{array}{c}
\omega_{1} u_{1} \\
\omega_{2} u_{2}
\end{array}\right)+\left(\begin{array}{cc}
\sigma_{1}^{2} \omega_{1} u_{1}^{2} & 0 \\
0 & \sigma_{2}^{2} \omega_{2} u_{2}^{2}
\end{array}\right) \\
=q_{1} u_{1}^{2}-q_{2} u_{2}^{2}+\left(\omega_{2}\left(b_{1} y_{2}-b_{2}\right)-\omega_{1} x_{2} a_{2}\right) u_{1} u_{2} .
\end{gathered}
$$

If the relations from the proposition take place, then we get $d V(t, u)=-u(t)^{T} Q u(t)$. The matrix $Q$ is symmetric and positive defined and has positive eigenvalues $r_{1}=q_{1}$ and $r_{2}=q$. Let $q_{m}$ be $q_{m}=$ $\min \left\{\mathrm{q}_{1}, \mathrm{q}_{2}\right\}$. It results that

$$
L V(t, u) \leq-q_{m}\|u(t)\|^{2},
$$

and so the equilibrium point is asymptotically stable in square mean. Let us choose the same parameters values for $a_{1}, a_{2}, b_{1}, b_{2}, b_{3}$ as on the simulation of Lyapunov exponents. We use Maple 12 software for the implementation of the second order Euler method. We observe from the following graphics that the solution trajectories represent the stable characteristic, which validate our theoretical discussion for the system of differential equation (25), for the equilibrium point $P_{2}$ (Figure 20-Figure 23). 


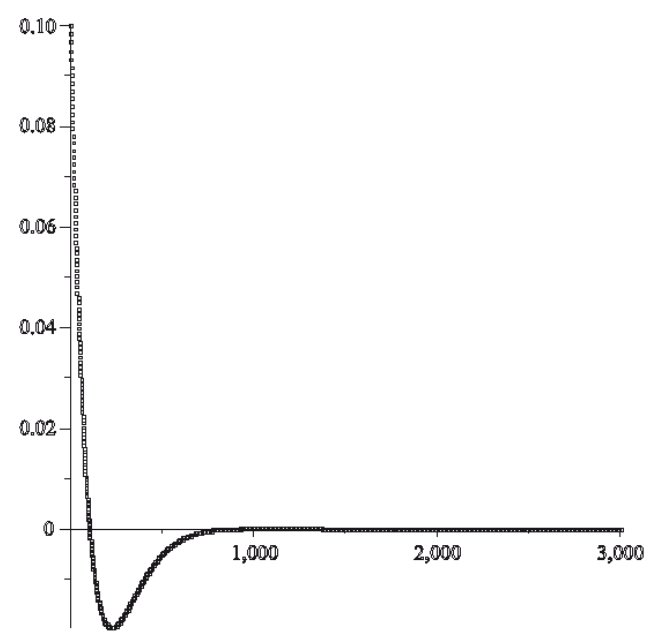

Figure 20: $(\mathrm{n}, \mathrm{x}(\mathrm{n}))$ in $\mathrm{P}_{2}$ for ODE (5)

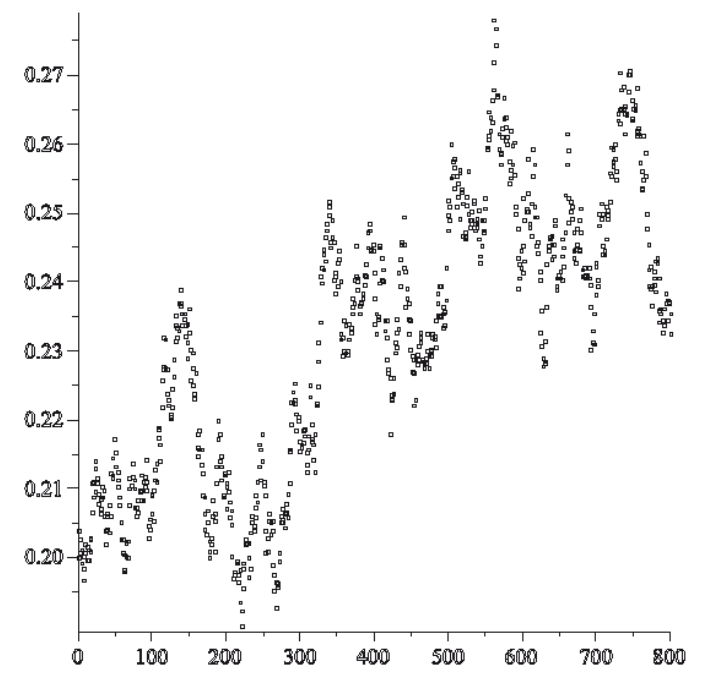

Figure 21: (n, $\mathrm{x}(\mathrm{n}, \omega))$ in $\mathrm{P}_{2}$ for SDE (26)
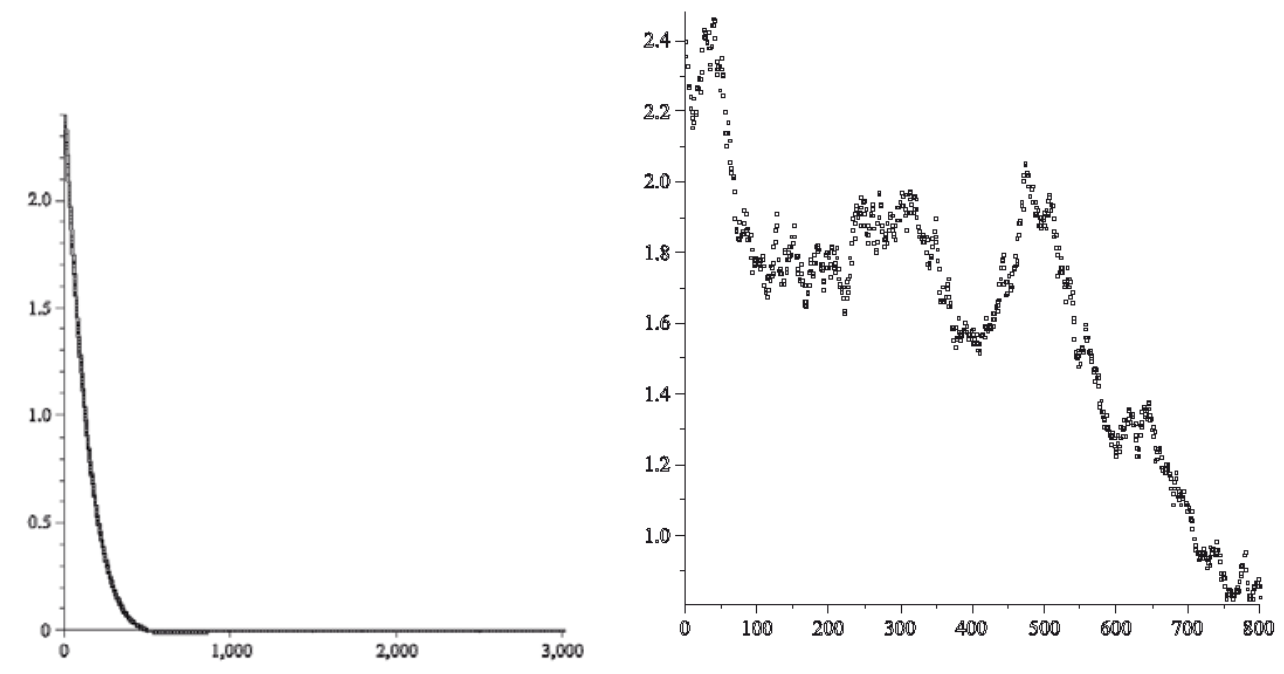

Figure 22: $(\mathrm{n}, \mathrm{x}(\mathrm{n}))$ in $\mathrm{P}_{2}$ for ODE (5) 
We will consider the study for the Bell model, in the equilibrium point $P_{2}$ from stability point of view. We will calculate and implement the second order moments associated to stochastic dynamical system (26). The characteristic function for differential equation for square root mean is given by (12)

$$
f(\lambda)=\left(\begin{array}{ccc}
2 \lambda-2 a_{11}-\sigma_{1}^{2} & 0 & 2 a_{12} \\
0 & 2 \lambda-2 a_{22}-\sigma_{2}^{2} & 2 a_{21} \\
a_{21} & a_{12} & 2 \lambda-a_{11}-a_{22}-\sigma_{1} \sigma_{2}
\end{array}\right),
$$

with

$$
\begin{gathered}
a_{11}=0, \\
a_{12}=-a_{2} \frac{a_{1} b_{3}-a_{2} b_{4}}{a_{1} b_{1}-a_{2} b_{2}} . \\
a_{22}=b_{1} \frac{a_{1} b_{3}-a_{2} b_{4}}{a_{1} b_{1}-a_{2} b_{2}}-b_{3} .
\end{gathered}
$$

If we consider $\sigma_{1}=-2$ and $\sigma_{2}=1$, the equilibrium point $P_{2}$ is stable, as the eigenvalues of the matrix $A$ in $P_{2}$ have negative real parts, but the moments of the second order are unstable, as it has a positive solution. The orbits of the stochastic Bell model, for specific values of $\sigma_{1}$ and $\sigma_{2}$ are represented in the following graphics (Figure 24 and Figure 25).
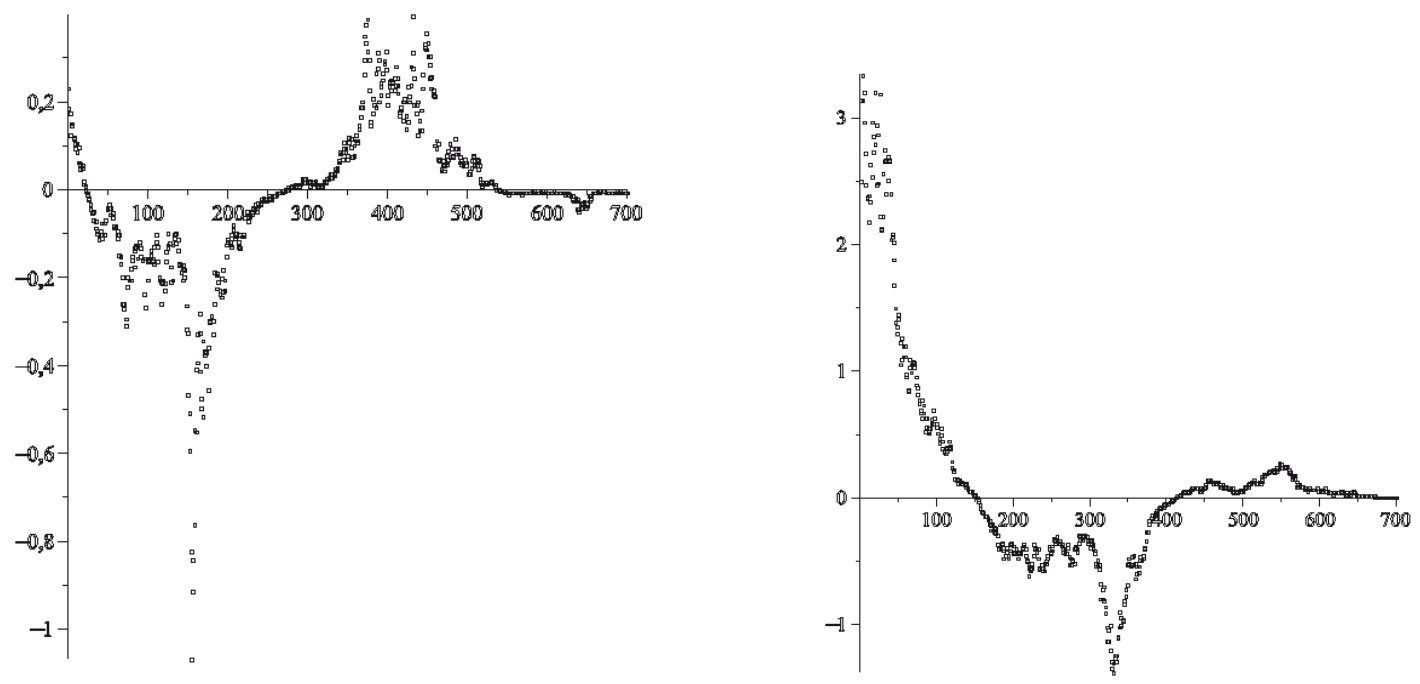
Figure 24: $\left(\mathrm{n}, \mathrm{y}_{1}(\mathrm{n}, \omega)\right)$

Figure 25: $\left(\mathrm{n}, \mathrm{y}_{2}(\mathrm{n}, \omega)\right)$

The orbits of the moments of the second order, $\left(n, R_{11}(n)\right)$, $\left(n, R_{22}(n)\right)$, are represented in Figure 26, Figure 27 and Figure 28, and the orbits for dispersions, $(n, D(1,1)(n))$, and $(n, D(2,2)(n))$, are represented in Figure 29 and Figure 30.
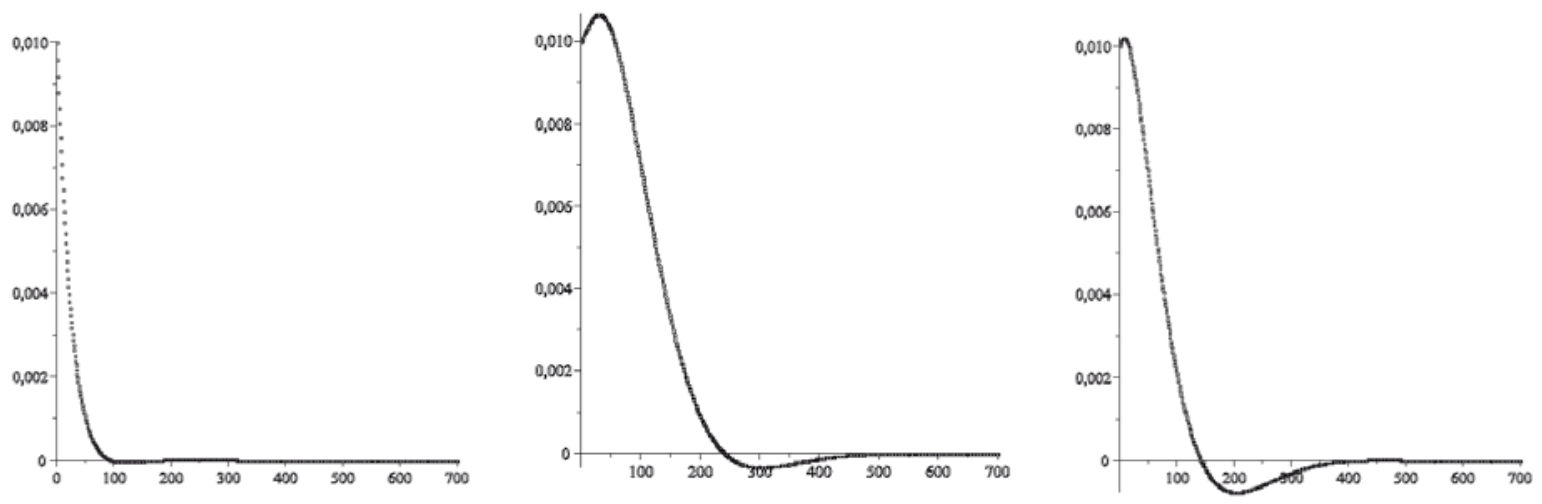

Figure 26: $\left(\mathrm{n}, \mathrm{R}_{11}(\mathrm{n})\right)$

Figure 27: (n, $\left.\mathrm{R}_{22}(\mathrm{n})\right)$

Figure 28: $\left(\mathrm{n}, \mathrm{R}_{12}(\mathrm{n})\right)$
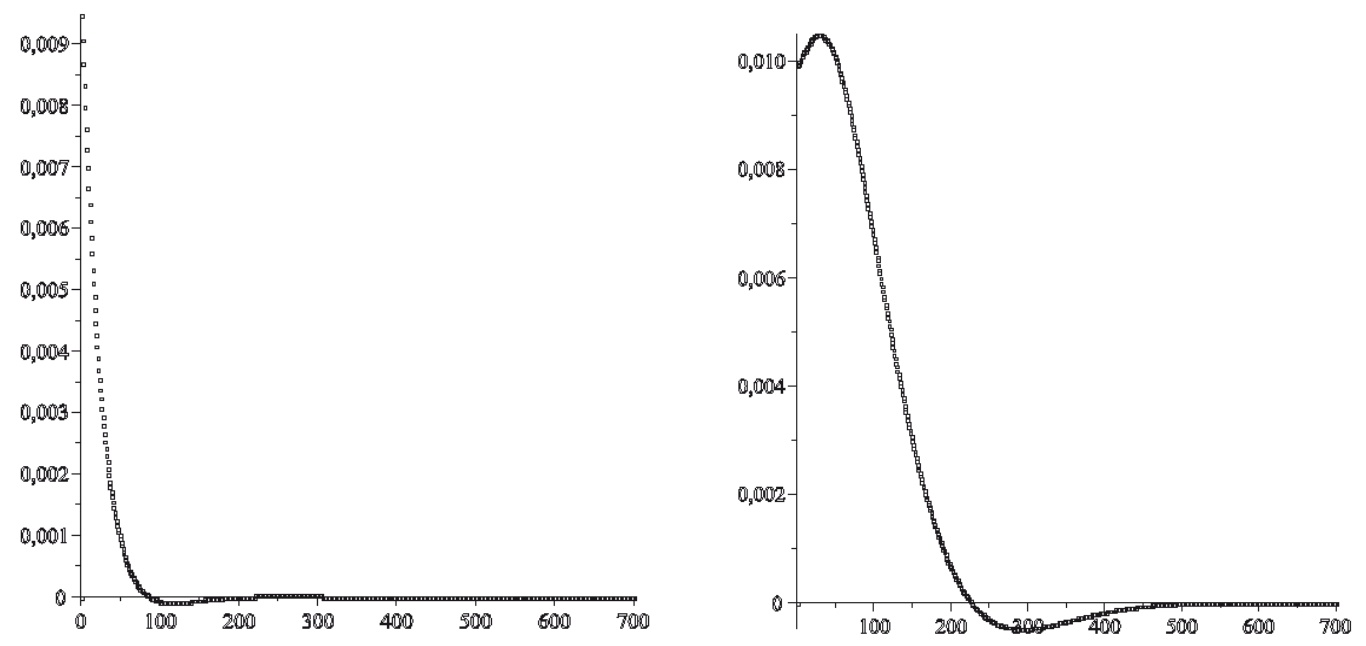

Figure 29: (n,D(1,1)(n))

Figure 30: (n,D(2,2)(n)) 


\section{Conclusions}

In this paper we have focused on two important tumor-immune systems, presented from stochastic point of view: a Kuznetsov-Taylor model and Bell model, that belongs to a general family of tumor-immune stochastic systems. We have determined the equilibrium points and we have calculated the Lyapunov exponents. These exponents help us to decide whether the stochastic model is stable or not. We have also proved stochastic stability by constructing a proper Lyapunov function, under a well chosen conditions. Another manner for verifying the stability is given by calculating the moments of the second order. We determined the differential equations for the mean values and square mean values and we have investigated the solution behavior. All our results were also proved using graphical implementation. For numerical simulations we have used the second order Euler scheme and the implementation of all those algorithms was done in Maple 12. In a similar way can be studied other models that derive from model given by (5). The model given by the SDE (20) allows the control of the system given by the ODE (1), with a stochastic process.

\section{Bibliography}

[1] Tan, W. Y., Chen, C.W. Cancer stochastic models. Encyclopedia of Statistical Sciences II, 2006, Published online.

[2] Albano, G.; Giorno, V. A stochastic model in tumor growth. J Theor Biol, 2006, 242, 329-336.

[3] Ferrante, L.; Bompadre, S.; Possati, L.; Leone, L. Parameter estimation in a Gompertzian stochastic model for tumor growth. Biometrics, 2000, 56, 1076-1081.

[4] Kuznetsov, V. A.; Makalkin, I. A.; Taylor, M. A.; Perelson, A. S. Nonlinear dynamics of immunogenic tumors: parameter estimation and global bifurcation analysis. Bull Math Biol, 1994, 56, 295-321.

[5] Jedrzejewski, F., Brochard, D. Lyapunov exponents and stability in stochastic dynamical structures. ASME-PUBLICATIONS-PVP, 2000.

[6] Schurz, H. Moment contractivity and stability exponents of nonlinear stochastic dynamical systems. IMA Print Series 1656, 1999. 
[7] Sotolongo-Costa, O., Morales-Molina, L., Rodriguez-Perez,D., Antoranz, J. C., ChaconReyes, M. Behavior of tumors under nonstationary therapy. Physica D, 2003, 178, 242253.

[8] d'Onofrio, A. A general framework for modeling tumor-immune system competition and immunotherapy: Mathematical analysis and biomedical inferences,. Physica D, 2005, 208, 220-235.

[9] Volterra, V. Variations and fluctuations of the number of individuals in animal species living together. 1931.

[10] Stepanova, N. V. Course of the immune reaction during the development of a malignant tumor. Biophysics, 1980, 24, 917-923.

[11] de Vladar, H. P.; Gonzalez, J. A. Dynamic response of cancer under the influence of immunological activity and therapy. $J$ Theor Biol, 2004, 227, 335-348.

[12] Wheldon, T. E. Mathematical Models in Cancer Research. 1988.

[13] Marusic, M.; Bajzer, Z.; Freyer, J. P.; Vuk-Pavlovic, S. Analysis of growth of multicellular tumour spheroids by mathematical models. Cell Prolif, 1994, 27, 73-94.

[14] S. Sahooa, A. S., S.F.C. Shearer Dynamics of Gompertzian tumour growth under environmental fluctuations. Physica A, 2010, 389, 1197-1207.

[15] Mackey, M. C.; Nechaeva, I. G. Solution moment stability in stochastic differential delay equations. Phys Rev E Stat Phys Plasmas Fluids Relat Interdiscip Topics, 1995, 52, 3366-3376.

[16] Oseledec, V. I. A multiplicative Ergodic theorem, Lyapunov characteristic numbers for dynamical systems. Trans. Moscow Math. Soc., 1968, 19, 197-231. 